\title{
Rol de los antiespasmódicos en el manejo del síndrome de intestino irritable (SII)
}

\section{The role of antispasmodics in managing irritable bowel syndrome}

Valeria Atenea Costa Barney, ${ }^{*}$ Alan Felipe Ovalle Hernández. ${ }^{1}$

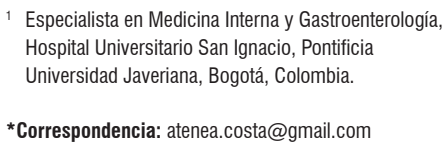

*Correspondencia: atenea.costa@gmail.com

\begin{abstract}
Resumen
El uso de los antiespasmódicos forma parte de la piedra angular del tratamiento en el síndrome de intestino irritable (SII), independientemente del subtipo. Consideramos relevante hacer una revisión de los medicamentos antiespasmódicos disponibles actualmente en Colombia, los cuales son usados crónicamente, de manera frecuente, en esta enfermedad.
\end{abstract}

\section{Palabras clave}

Antiespasmódicos, síndrome de intestino irritable, bromuro de pinaverio, bromuro de otilonio, mebeverina, trimebutina.

\begin{abstract}
Although antispasmodics are the cornerstone of treating irritable bowel syndrome, there are a number of antispasmodic medications currently available in Colombia. Since they are frequently used to treat this disease, we consider an evaluation of them to be important.

Keywords

Antispasmodic, irritable bowel syndrome, pinaverium bromide, otilonium bromide, Mebeverin, trimebutine.
\end{abstract}

\section{INTRODUCCIÓN}

El síndrome del intestino irritable (SII) es uno de los trastornos funcionales gastrointestinales crónicos más frecuentes; está caracterizado por la presencia de dolor abdominal recurrente asociado a alteraciones del ritmo de las deposiciones, ya sea en forma de estreñimiento, de diarrea, o de ambas, en donde la hinchazón y la distensión abdominal son muy frecuentes (1).

El SII se divide, según el tipo de alteración del hábito digestivo, en SII con predominio de estreñimiento (20$30 \%$ de los pacientes) y SII con predominio de diarrea (20$30 \%$ de los pacientes); cuando se combinan ambos trastornos, estreñimiento y diarrea, se habla de SII de tipo mixto (hasta $45 \%$ de los pacientes), y de SII de tipo indetermi- nado, cuando el patrón de las deposiciones es intermedio y no puede clasificarse como diarrea ni estreñimiento. Es de resaltar que la presencia de dolor abdominal más de una vez a la semana, así como la relación temporal del dolor con la defecación, es lo que teóricamente diferencia al SII de tipo del estreñimiento funcional $(2,3)$.

De acuerdo con los criterios de Roma IV, el SII se diagnostica por la presencia de dolor abdominal recurrente que debe estar presente al menos un día a la semana, con dos o más de las siguientes características: se asocia a la defecación; está relacionado con un cambio en la frecuencia de las deposiciones; y está relacionado con un cambio en la consistencia de las deposiciones. En cuanto a los requerimientos de duración de las molestias, hay que tener en cuenta que los criterios deben cumplirse durante los últimos tres 
meses, y los síntomas, haber comenzado un mínimo de seis meses antes del diagnóstico $(3,4)$.

No se conocen explicaciones estructurales o anatómicas que expliquen la fisiopatología del SII, y se desconoce la causa exacta, aunque se han propuesto varios mecanismos. La alteración de la motilidad gastrointestinal puede contribuir al cambio en el hábito intestinal informado por algunos pacientes, y la combinación de espasmo del músculo liso, hipersensibilidad visceral y anomalías del procesamiento del dolor central pueden explicar el dolor abdominal, que es una parte esencial del complejo sintomático (5).

Se estima que afecta al $11 \%$ de la población mundial; en Europa, Asia y Estados Unidos la prevalencia varía del 10-20\%, con baja prevalencia en el sur de Asia (7\%), siendo la más alta en Suramérica (21\%), y en los países occidentales es dos veces más frecuente en las mujeres (6). El SII tiene un impacto importante en la calidad de vida relacionada con la salud, lo que resulta en una menor productividad laboral, un elevado ausentismo y un mayor uso y costos de la atención médica. En 2005, en Estado Unidos, se estimaron costos médicos directos atribuidos al SII entre USD 1,5-10 mil millones por año (1). Cabe resaltar que el SII también puede afectar la relación médico-paciente, ya que el control ineficaz de los síntomas puede disminuir la credibilidad de los médicos y estimular al paciente a buscar opiniones adicionales (7).

Dentro del manejo del SII existe un grupo heterogéneo de medicamentos llamados antiespasmódicos o espasmolíticos, que pueden actuar como relajantes musculares lisos directos (papaverina, mebeverina, aceite de menta), agentes anticolinérgicos (butilescopolamina, hioscina, bromuro de cimetropio, pirenzepina) y bloqueadores de los canales de calcio (citrato de alverina, bromuro de otilonio, bromuro de pinaverio). Estos medicamentos se han utilizado en la terapia para el SII durante décadas, cuyo objetivo es reducir los síntomas condicionados por la defecación al aumentar el tiempo de tránsito colónico, adicionalmente, mejorar la consistencia de las heces y reducir la frecuencia de las deposiciones (2). La acción farmacológica de estos agentes no siempre es clara, y los mecanismos a menudo son mixtos. Sin embargo, los metaanálisis realizados en estudios que comparan antiespasmódicos con placebo $\mathrm{u}$ otros tratamientos han confirmado de manera uniforme los efectos positivos de estos fármacos, y sus perfiles de efectos secundarios han sido excelentes (8).

\section{FISIOPATOLOGÍA}

Se considera que la patogénesis del SII es multifactorial; el antecedente de infecciones gastrointestinales, flora colónica o bacteriana del intestino delgado alterada, el aumento de la permeabilidad intestinal y la activación inmune pueden desempeñar un papel en el desarrollo de la enfermedad (9). Es conocido que las señales del tracto gastrointestinal se procesan en el cerebro, lo que a su vez puede influir en la motilidad, la secreción y la función inmune. El eje cerebro-intestino es esencial para la regulación del sistema gastrointestinal, y su alteración estructural o funcional puede conducir al desarrollo de trastornos como el SII (10), por lo que los factores psicológicos y el estrés crónico también pueden estar involucrados en el desencadenamiento de los síntomas (11).

La motilidad intestinal anormal y la hipersensibilidad visceral siguen siendo los principales factores en la patogénesis de la enfermedad (12). Se ha descrito que los factores intraluminales, como las proteasas de serina, pueden aumentar la permeabilidad colónica en pacientes con SII mediante la activación del receptor-2 activado por la proteasa, lo que produce hipersensibilidad visceral (13). A su vez, se ha demostrado que las cisteínas proteasas luminales aumentan la permeabilidad colónica a través de la degradación de proteínas de unión, lo que produce hipersensibilidad visceral posiblemente secundaria a la microinflamación local (14). La activación inmune de la mucosa colónica fue significativamente mayor en el SII que en controles sanos (15). Los mastocitos se han visto implicados en el desarrollo del SII: el número de mastocitos en la mucosa colónica y su liberación de tripsina e histamina se incrementaron marcadamente en pacientes con SII en comparación con los controles (16). Además, la presencia de los mastocitos en las proximidades de las terminaciones nerviosas se ha correlacionado significativamente con la gravedad y frecuencia del dolor/malestar abdominal en pacientes con SII. Las células enterocromafines también desempeñan un papel importante en el desarrollo de la hipersensibilidad visceral, ya que producen y liberan serotonina, que activa los receptores $5-\mathrm{HT}_{3}$ ubicados en las neuronas sensitivas aferentes (17).

Las anormalidades en los patrones de motilidad colónica se caracterizan por la hiperreactividad, debido a un aumento prolongado de la actividad motora colónica después de las comidas, un aumento de la actividad motora en respuesta a factores estresantes o colecistoquinina (CCK), y una mayor actividad motora en respuesta a la distensión abdominal (18).

La hipersensibilidad visceral por sí sola no es dolorosa, pero puede provocar dolor abdominal en pacientes con SII por el efecto de un estímulo intenso, como una contracción exagerada del colon (19). Sin embargo, no se ha podido establecer una relación clara entre la hipersensibilidad visceral y los trastornos de la motilidad; estos dos factores se han considerado generalmente independientes, $\mathrm{y}$ ambos requieren un tratamiento eficaz (20). 


\section{Canales de calcio dependientes de voltaje}

Los canales de calcio dependientes de voltaje desempeñan un papel fundamental en el intestino, así como en el manejo farmacológico del SII, ya que son canales iónicos que median la afluencia de calcio en respuesta a la despolarización de la membrana, y regulan los procesos intracelulares, como la contracción, la secreción y la neurotransmisión en una variedad de células (21). Los canales de calcio se clasifican por sus propiedades y su farmacología (22), entre los que se destacan los canales de calcio tipo L (larga duración), que son canales de gran conductancia que producen una corriente de despolarizaciones de larga duración, y son inhibidos por los derivados de dihidropiridina (DHP) (23). Las corrientes asociadas a este tipo de canales son importantes para las células musculares y endocrinas, en las cuales se media la contracción y la secreción de sustancias (21).

Las corrientes de tipo $\mathrm{N}$ (neuronal) también son duraderas, pero requieren potenciales fuertemente negativos para la eliminación completa de la inactivación, y fuertes despolarizaciones para la activación (23). En las células de Purkinje, se han identificado otros tres canales. Las corrientes de tipo $\mathrm{P}$ están bloqueadas por bajas concentraciones de $\omega$-agatoxina, mientras que el tipo $\mathrm{Q}$ solo responde a altas concentraciones. Las corrientes residuales, que eran resistentes a todos los bloqueadores de calcio conocidos en el momento de su descubrimiento, se denominaron tipo $\mathrm{R}$ (resistente). El último grupo de canales de calcio dependientes de voltaje, el tipo $\mathrm{T}$ (transitorio), se caracteriza por una conductancia pequeña y transitoria activada por despolarizaciones débiles (23). Estas corrientes son responsables de la modulación del potencial de acción y del rendimiento de las actividades de marcapasos.

El uso médico de los antagonistas del calcio comenzó en la década de 1980 con los antagonistas del tipo DHP, que bloquean los canales de tipo L en el tratamiento de la hipertensión al explotar sus propiedades como vasodilatadores (22). Los antagonistas del calcio no tienen efecto sobre el músculo esquelético; sin embargo, influyen levemente en el músculo cardíaco al disminuir la actividad y la conducción del marcapasos. Sobre la base de las alteraciones de la motilidad gastrointestinal en el SII, los antagonistas del calcio utilizados para las enfermedades cardiovasculares parecen ser opciones potenciales para aliviar los síntomas al relajar los músculos lisos del colon. Por lo tanto, a finales de la década de 1980, se propuso el uso de la nicardipina para el tratamiento del SII, en función de sus propiedades espasmolíticas (24). No obstante, los efectos secundarios cardiovasculares han limitado seriamente la aplicación de dichos antagonistas del calcio, lo cual condujo a los investigadores a identificar sustancias que actuaran selectivamente en el tracto gastrointestinal.

\section{MEDICAMENTOS ANTIESPASMÓDICOS}

Estos medicamentos actuán inhibiendo la acción de la acetilcolina en los receptores muscarínicos, o mediante el bloqueo de los canales de calcio en el músculo liso gastrointestinal. Como clase, los antiespasmódicos se han utilizado en el tratamiento del SII durante muchos años, sobre la base de que un subgrupo de pacientes con SII tiene una contractilidad anormal del músculo liso gastrointestinal y un tránsito gastrointestinal alterado, lo cual contribuye a la presencia de dolor y a las alteraciones del hábito intestinal (25).

\section{Bromuro de otilonio}

Su estructura está constituida principalmente por amonio cuaternario, por lo cual es débilmente absorbible del tracto gastrointestinal. En estudios experimentales se evidencia su acúmulo en las paredes del tracto gastrointestinal luego de su administración oral, con una excreción casi completa en las heces (25). El bromuro de otilonio no solo bloquea los canales de calcio tipo $\mathrm{L}$ y tipo $\mathrm{T}$, sino también a los receptores muscarínicos M1, M2, M4 y M5, en donde el antagonismo de señales de calcio acopladas a M3 en las células de las criptas colónicas humanas sugirió una acción antisecretora en pacientes con SII de tipo diarrea. Además, debido al antagonismo de los receptores de taquicinina neuroquinina-2 (NK-2), no solo causa espasmólisis sino que también reduce la transmisión aferente sensorial periférica al sistema nervioso central, posiblemente contribuyendo a su mayor eficacia $(26,27)$. Estos efectos sugieren que el otilonio podría ser efectivo para reducir los dos síntomas principales del SII: espasmos y dolor abdominal (2).

En el manejo del dolor abdominal en el SII, el bromuro de otilonio fue evaluado en un ensayo clínico en el que los pacientes diagnosticados según los criterios de Roma II recibieron $40 \mathrm{mg}$ de bromuro de otilonio tres veces al día o placebo durante 15 semanas después de la aleatorización. Se encontró que redujo la frecuencia del dolor y la distensión abdominal, y que mejoró, a su vez, la frecuencia de las deposiciones y la evaluación global de síntomas de los pacientes, en comparación con el placebo. El resultado destacado de este estudio fue la eficacia del otilonio para reducir de manera importante la frecuencia del dolor abdominal de más de la mitad de los días a menos de un día por semana, en comparación con los 1-3 episodios persistentes en el grupo placebo. Durante el período de seguimiento de 10 semanas, después de finalizar el tratamiento, la probabilidad de recurrencia de los síntomas fue significativamente mayor en el grupo de placebo que en el grupo de bromuro de otilonio $(28,29)$. Este hallazgo podría explicarse por la persistencia prolongada del bromuro de otilonio en la pared del colon debido a sus propiedades lipofílicas (25). 
Dentro de los efectos secundarios más comunes de reportados asociados al uso de bromuro de otilonio se encuentran la sequedad de la boca, las náuseas, y el mareo, que pueden ser causados por el antagonismo muscarínico periférico y central, y podrían explicarse por la capacidad conocida de otilonio para unirse a receptores muscarínicos (27).

El bromuro de otilonio ha sido evaluado en 5 estudios aleatorizados controlados (29-33), en los cuales se incluyeron 791 pacientes con evidencia de un efecto beneficioso (razón de riesgo $(\mathrm{RR})=0,70$; intervalo de confianza (IC) del $95 \% 0,54-0,90)$ y el número necesario a tratar (NNT) de 5 (IC $44 \%, p=0,13$ ), pero una heterogeneidad limítrofe entre los resultados del estudio $\left(I^{2}=44 \%, p=0,13\right)$. Con base en las recomendaciones de las guías de práctica clínica publicadas por la Asociación Colombiana de Gastroenterología (ACG), se da una recomendación fuerte a favor para el uso de bromuro de otilonio para incrementar la frecuencia de mejoría global de síntomas en pacientes con SII (calidad de evidencia baja).

\section{Bromuro de pinaverio}

Es derivado del amonio cuaternario, se absorbe poco, y tiene efectos farmacológicos pronunciados en el tracto gastrointestinal en lugar del sistema cardiovascular (34). Tiene una baja tasa de absorción del tracto gastrointestinal, caracterizada por la excreción hepatobiliar (35). Sus efectos son muy similares a los de los bloqueadores de los canales de calcio de tipo L establecidos (nitrendipina, diltiazem), ya que reduce la fase de meseta de las ondas lentas, lo que inhibe la afluencia de calcio y evita las contracciones subsecuentes (36).

Además, se ha demostrado que inhibe la respuesta contráctil en el músculo liso del colon de perros y ratas de la acetilcolina, neurotransmisor de los nervios colinérgicos intrínsecos (36). De manera similar, en las células de músculo liso del colon aisladas del colon humano normal o inflamado, inhibe la contracción inducida por diferentes agonistas (CCK) (37). Se ha demostrado la participación de las neuronas aferentes sensoriales en el SII, lo que también podría explicar la eficacia del bromuro de pinaverio en el tratamiento de las dos características principales del SII: los trastornos de la motilidad y la hipersensibilidad intestinal.

En un estudio piloto realizado en 12 pacientes con SII, se detectó la motilidad colónica mediante electromiografía de superficie durante un período de ayuno de dos horas y un período posprandial de dos horas después de una comida estándar, antes y después de 10 días de tratamiento con 50 $\mathrm{mg}$ de bromuro de pinaverio tres veces al día. Los síntomas principales, como dolor abdominal, distensión abdominal y alteración del hábito intestinal, comenzaron a mejorar el día 4 del tratamiento. Los patrones anormales de motilidad colónica (mayor frecuencia y amplitud de contracción y alteración del ritmo en la actividad motora) disminuyeron después de 10 días de tratamiento. En una continuación de este estudio, los autores estudiaron 22 pacientes con SII y 7 controles sanos (38). Los controles sanos no recibieron tratamiento, pero sirvieron como controles para las mediciones electromiográficas. El protocolo de estudio fue como se describió anteriormente, excepto por la duración de la terapia con bromuro de pinaverio, que se extendió a 14 días. Los resultados mostraron un aumento de los parámetros de motilidad colónica en ayunas y posprandial en pacientes con SII en comparación con los controles, que se redujo efectivamente en 14 días de terapia con bromuro de pinaverio. El dolor y la distensión abdominales también mejoraron significativamente con el tratamiento.

Dentro de los efectos adversos descritos se ha evidenciado la presencia de hipersensibilidad, angioedema, estreñimiento, somnolencia, disfagia, dolor epigástrico, eritema, cefalea, náuseas, prurito, vértigo, vómito y xerostomía. El uso del bromuro de pinaverio generalmente se ha considerado seguro; sin embargo, su uso está contraindicado en mujeres embarazadas. Los estudios de reproducción animal son insuficientes y la información de embarazos humanos no está disponible, lo que planta un riesgo teórico de efectos neurológicos (sedación e hipotonía) en los recién nacidos si se usa pinaverio al final del embarazo, aunque no se han informado casos similares.

El bromuro de pinaverio ha sido evaluado en 4 estudios (39-42), en 615 pacientes, en los que se encontró un efecto estadísticamente significativo en la mejoría de los síntomas del SII (RR = 0,56; IC del $95 \% 0,38-0,82)$ con un NNT de 4 (IC del $95 \%$ 3-6), y se presentó heterogeneidad estadísticamente significativa $\left(I^{2}=61 \%, p=0,05\right)$. Basados en las recomendaciones de las guías de práctica clínica publicadas por la ACG, se da una recomendación fuerte a favor para el uso de bromuro de pinaverio para disminuir el dolor abdominal en pacientes con SII (calidad de evidencia baja).

\section{Trimebutina}

El modo de acción en el tracto gastrointestinal del ácido 3,4,5-trimetoxibenzoico 2 (dimetilamino)-2-fenilbutilester, que es la trimebutina, es multifacético. Tiene una actividad espasmolítica única y muestra una actividad agonista no selectiva significativa para los receptores opioides intestinales $\mu, \kappa$ y $\delta$. Se ha informado que la trimebutina induce prematuramente la fase III del complejo motor migratorio (CMM) en el intestino, y también se ha demostrado que modula la sensibilidad visceral. Por otra parte, es probable que actúe sobre las células intersticiales de Cajal, claves en la iniciación y la regulación de la motilidad gastrointestinal, así como en los músculos lisos y los nervios entéricos. 
Algunos estudios han informado que la trimebutina actúa como regulador del canal de $\mathrm{Ca}^{2+}$ y K $\mathrm{K}^{+}$en el intestino (43).

Se realizó un estudio clínico multicéntrico, aleatorizado, doble ciego, sin inferioridad para comparar fenoverina con trimebutina. Los sujetos fueron asignados al azar para recibir fenoverina ( $100 \mathrm{mg}$ tres veces al día) o trimebutina ( $150 \mathrm{mg}$ tres veces al día) durante 8 semanas. Un total de 197 pacientes fueron analizados. El criterio primario de evaluación fue la proporción de pacientes que tuvieron una reducción del $30 \%$ en el dolor abdominal medido por la escala de síntomas intestinales en la semana 8 , en comparación con la línea basal (44).

Los criterios de valoración fueron los cambios en la distensión abdominal, la diarrea, el estreñimiento, y los puntajes generales y de satisfacción general. En la semana 8, se demostró que la fenoverina no es inferior a la trimebutina (diferencia de tratamiento, 1,76\%; IC del $90 \%,-10,30$ 13,82; $p=0,81$ ); El 69,23 \% (54 de 78 pacientes) de los pacientes que tomaron fenoverina y el 67,47 \% (56 de 83 pacientes) de los pacientes que tomaron trimebutina mostraron una reducción del $30 \%$ en el dolor o malestar abdominal en comparación con la línea basal (44).

Dos revisiones sistemáticas informaron sobre el tratamiento con trimebutina en comparación con el placebo para pacientes con SII. Ambas revisiones sistemáticas mostraron una mejoría mayor en el dolor abdominal con el tratamiento con trimebutina en comparación con el placebo. Sin embargo, los resultados fueron estadísticamente significativos en una revisión sistemática con un RR de 1,32 y IC del $95 \%$ de 1,07 a 1,64 basados en tres ensayos controlados aleatorizados y no estadísticamente significativos en una revisión sistemática con un odds ratio (OR) de 1,28 y un IC del $95 \%$ de 0,53 a 3,14, según dos ensayos controlados aleatorizados (45).

No hubo una mejoría estadísticamente significativa en la evaluación global con trimebutina en comparación con el placebo: RR de 0,97; IC del 95 \%: 0,68-1,38 en una revisión sistemática basada en dos ensayos controlados aleatorizados, y OR 1,27; IC del 95 \%: 0,58-2,79 en una revisión sistemática con dos ensayos controlados aleatorizados. Una revisión sistemática reportó que no hubo diferencia estadísticamente significativa en los eventos adversos entre trimebutina y placebo: OR 0,62; IC del $95 \%$ : 0,20-1,88, basado en un ECA (45).

Un ensayo controlado aleatorizado informó que se observó una recuperación clínica en el 94,9 \% de los pacientes tratados con trimebutina y una recuperación espontánea en el 20,5 \% de los pacientes no tratados. Estos hallazgos se basaron en las respuestas de los padres, a quienes se les preguntó si su hijo tenía un alivio adecuado del dolor, y la incomodidad presentada del SII en los últimos siete días (45).
Un ensayo controlado aleatorizado que comparó trimebutina con mebeverina mostró que después de seis semanas de tratamiento hubo una mejoría estadísticamente significativa en los síntomas (dolor abdominal, consistencia y frecuencia de las heces y flatulencia) en comparación con los valores de referencia para cada fármaco ( $p$ que varía entre $<0,01 \mathrm{a}<0,05$ ). Sin embargo, no hubo una diferencia estadísticamente significativa en la mejoría de los síntomas entre los dos fármacos (los valores de $p$ oscilan entre $<0,23$ y $<0,71)$. En comparación con la línea basal, también se informaron una mejoría de los síntomas estadísticamente significativa con ambos fármacos después de una semana de tratamiento. La calidad de vida se evaluó con el cuestionario IBS-QoL y mejoró de manera estadísticamente significativa después del tratamiento con trimebutina o mebeverina $(p<0,05)$. Además, la mejoría con trimebutina fue estadísticamente mayor que con mebeverina $(p<0,05)$. Los autores declararon que no hubo diferencias en los eventos adversos entre los dos fármacos, sin embargo, no se presentaron datos cuantitativos (45).

Basados en las recomendaciones de las guías de práctica clínica publicadas por la ACG se da una recomendación débil a favor, que sugiere el uso de trimebituna para mejorar el dolor abdominal en pacientes con SII (calidad de evidencia baja).

\section{Mebeverina}

Es una beta-feniletilamina derivada de la reserpina, que tiene efectos relativamente específicos sobre las células del músculo liso, sin tener efectos tipo atropina en humanos. Bloquea directamente los canales de sodio e inhibe la acumulación de calcio intracelular. Es tres veces más potente que la papaverina en la inhibición del reflejo peristáltico ileal como se ha demostrado en experimentos animales en cerdos, sin embargo, otros estudios en animales sobre su farmacología muestran la ausencia de este efecto (2).

En un estudio temprano de Connell, la mebeverina endovenosa disminuyó toda la motilidad colónica sigmoidea, especialmente en sujetos hiperactivos, y tuvo menor o ningún efecto en sujetos hipoactivos. La mebeverina fue superior al placebo en un estudio de 12 semanas de tratamiento en pacientes con SII en términos de mejoría de los síntomas y bienestar general. Utilizando manometría ambulatoria prolongada en 12 pacientes con SII y 6 controles sanos, en comparación con un período de placebo, mebeverina no tuvo efectos significativos en el complejo motor interdigestivo del intestino delgado; por el contrario, se observó un mayor índice de motilidad de fase 2 tanto en SII de tipo diarrea como en los pacientes con SII de tipo estreñimiento, y el índice de motilidad de fase 3 también se vio afectado. Estas alteraciones en la actividad motora del 
intestino delgado por mebeverina sugieren posibles efectos espasmolíticos y procinéticos en pacientes con SII (2).

Con respecto al control de los síntomas en el SII en estudios sin placebo, se han obtenido resultados positivos. Una mejoría significativa se observó después de 6 semanas de tratamiento con las formas simples y sostenidas de mebeverina, con un número mínimo de eventos adversos. Al comparar el bromuro de pinaverio con la mebeverina en 91 pacientes con SII de tipo diarrea, las mejoras en el bienestar global fueron similares en los dos grupos, la frecuencia de defecación disminuyó notablemente, la consistencia de las deposiciones se formó bien en ambos grupos, y no se observaron efectos secundarios significativos. En un ensayo clínico que comparó los efectos de ramosteron, un receptor $5-\mathrm{HT}_{3}$ antagonista, con los de mebeverina en pacientes con SII de tipo diarrea, encontró que ambos tratamientos fueron igualmente efectivos en la reducción del dolor/incomodidad y la urgencia, con una mejoría de la puntuación de Bristol de la materia fecal y la frecuencia de las deposiciones en comparación con la línea basal (2).

Sin embargo, cuando los efectos de la mebeverina han sido comparados con placebo y con otro medicamento o cuando han sido medidos por el autocontrol, los resultados han sido controvertidos. Una revisión sistemática reciente, que incluyó ocho ensayos aleatorizados, reveló que la mejoría clínica y el alivio del dolor abdominal con el tratamiento con mebeverina no fueron estadísticamente significativos en comparación con el placebo. No se encontraron diferencias en la efectividad de 200 y $135 \mathrm{mg}$ de dosis de mebeverina. La tolerabilidad fue excelente, sin efectos adversos significativos. Del mismo modo, la mebeverina no tuvo efectos sobre el placebo en un estudio realizado en 135 pacientes con SII, cumpliendo los criterios de Roma III, que fueron reclutados de práctica general, cuando se comparó mebeverina, metilcelulosa y placebo con o sin la combinación de terapia cognitiva conductual (2). Sin embargo, en un estudio realizado en Londres, las sesiones de terapia cognitiva conductual fueron beneficiosas, además de la mebeverina, y los efectos persistieron hasta 6 meses después de la terapia, tanto en términos de alivio de síntomas como en la disminución de la discapacidad social y laboral. La depresión y la ansiedad predicen malos resultados en pacientes con SII tratados con mebeverina, y en casos de pacientes con alteraciones de comportamientos (por ejemplo, evitación), la combinación de mebeverina con terapia cognitiva conductual podría ser útil (2).

Basados en las recomendaciones de las guías de práctica clínica publicadas por la ACG, se da una recomendación débil a favor que sugiere el uso de mebeverina para el tratamiento de pacientes con SII (calidad de evidencia baja).

En conclusión, el efecto individual de los antiespasmódicos ha sido difícil de interpretar, dado que solo existe un pequeño número de estudios que evalúan cada medicamento; sin embargo, en estos se encontró que los antiespasmódicos son más efectivos que el placebo en el tratamiento del SII. De los medicamentos estudiados, el otilonio y el pinaverio son derivados cuaternarios del amonio, que son mal absorbidos en el tracto gastrointestinal, por tanto, actúan principalmente a nivel local por medio de la reducción de los efectos adversos de este grupo de medicamentos $y$, a su vez, reducen significativamente el riesgo de síntomas persistentes frente a placebo.

\section{REFERENCIAS}

1. Ford AC, Moayyedi P, Chey WD, Harris LA, Lacy BE, Saito YA, et al. American College of Gastroenterology Monograph on Management of Irritable Bowel Syndrome. Am J Gastroenterol. 2018 Jun;113(Suppl 2):1-18. doi: 10.1038/s41395-018-0084-x.

2. Annaházi A, Róka R, Rosztóczy A, Wittmann T. Role of antispasmodics in the treatment of irritable bowel syndrome. World J Gastroenterol. 2014 May 28;20(20):603143. doi: 10.3748/wjg.v20.i20.6031.

3. Lacy BE, Mearin F, Chang L, Chey WD, Lembo AJ, Simren $\mathrm{M}$, et al. Bowel Disorders. Gastroenterology. 2016 May 1;150(6):1393-1407.e5. doi: 10.1053/j.gastro.2016.02.031.

4. Longstreth GF, Thompson WG, Chey WD, Houghton LA, Mearin F, Spiller RC. Functional bowel disorders. Gastroenterology. 2006 Apr;130(5):1480-91. doi: 10.1053/j.gastro.2005.11.061.

5. Martínez-Vázquez MA, Vázquez-Elizondo G, GonzálezGonzález JA, Gutiérrez-Udave R, Maldonado-Garza HJ, Bosques-Padilla FJ. Effect of antispasmodic agents, alone or in combination, in the treatment of Irritable Bowel Syndrome: systematic review and meta-analysis. Rev Gastroenterol Mex. 2012 Apr-Jun;77(2):82-90. doi: 10.1016/j.rgmx.2012.04.002.

6. Janssen HA, Borghouts JA, Muris JW, Metsemakers JF, Koes BW, Knottnerus JA. Health status and management of chronic non-specific abdominal complaints in general practice. Br J Gen Pract. 2000 May;50(454):375-9.

7. Ford AC, Talley NJ, Spiegel BM, Foxx-Orenstein AE, Schiller L, Quigley EM, et al. Effect of fibre, antispasmodics, and peppermint oil in the treatment of irritable bowel syndrome: systematic review and meta-analysis. BMJ. 2008 Nov 13;337:a2313. doi: 10.1136/bmj.a2313.

8. Poynard T, Regimbeau C, Benhamou Y. Meta-analysis of smooth muscle relaxants in the treatment of irritable bowel syndrome. Aliment Pharmacol Ther. 2001 Mar;15(3):35561. doi: 10.1046/j.1365-2036.2001.00937.x.

9. Neal KR, Hebden J, Spiller R. Prevalence of gastrointestinal symptoms six months after bacterial gastroenteritis and risk factors for development of the irritable bowel syndrome: postal survey of patients. BMJ. 1997 Mar 15;314(7083):77982. doi: 10.1136/bmj.314.7083.779. 
10. Fichna J, Storr MA. Brain-Gut Interactions in IBS. Front Pharmacol. 2012 Jul 5;3:127. doi: 10.3389/ fphar.2012.00127.

11. Levy RL, Olden KW, NaliboffBD, BradleyLA, Francisconi C, Drossman DA, Creed F. Psychosocial aspects of the functional gastrointestinal disorders. Gastroenterology. 2006 Apr;130(5):1447-58. doi: 10.1053/j.gastro.2005.11.057.

12. Bouin M, Plourde V, Boivin M, Riberdy M, Lupien F, Laganière $M$, et al. Rectal distention testing in patients with irritable bowel syndrome: sensitivity, specificity, and predictive values of pain sensory thresholds. Gastroenterology. 2002 Jun; 122(7):1771-7. doi: 10.1053/gast.2002.33601.

13. Gecse K, Róka R, Ferrier L, Leveque M, Eutamene H, Cartier $\mathrm{C}$, et al. Increased faecal serine protease activity in diarrhoeic IBS patients: a colonic lumenal factor impairing colonic permeability and sensitivity. Gut. 2008 May;57(5):591-9. doi: 10.1136/gut.2007.140210.

14. Annaházi A, Ferrier L, Bézirard V, Lévêque $M$, Eutamène $H$, Ait-Belgnaoui A, et al. Luminal cysteine-proteases degrade colonic tight junction structure and are responsible for abdominal pain in constipation-predominant IBS. Am J Gastroenterol. 2013 Aug;108(8):1322-31. doi: 10.1038/ ajg.2013.152.

15. Ahn JY, Lee KH, Choi CH, Kim JW, Lee HW, Kim JW, et al. Colonic mucosal immune activity in irritable bowel syndrome: comparison with healthy controls and patients with ulcerative colitis. Dig Dis Sci. 2014 May;59(5):1001-11. doi: 10.1007/s10620-013-2930-4.

16. Barbara G, Stanghellini V, De Giorgio R, Cremon C, Cottrell GS, Santini D, et al. Activated mast cells in proximity to colonic nerves correlate with abdominal pain in irritable bowel syndrome. Gastroenterology. 2004 Mar;126(3):693702. doi: 10.1053/j.gastro.2003.11.055.

17. Dunlop SP, Jenkins D, Neal KR, Spiller RC. Relative importance of enterochromaffin cell hyperplasia, anxiety, and depression in postinfectious IBS. Gastroenterology. 2003 Dec;125(6):1651-9. doi: 10.1053/j.gastro.2003.09.028.

18. Lind CD. Motility disorders in the irritable bowel syndrome. Gastroenterol Clin North Am. 1991 Jun;20(2):279-95.

19. Clavé P. Treatment of IBS-D with 5-HT3 receptor antagonists vs spasmolytic agents: similar therapeutical effects from heterogeneous pharmacological targets. Neurogastroenterol Motil. 2011 Dec;23(12):1051-5. doi: 10.1111/j.13652982.2011.01808.x.

20. Kanazawa M, Palsson OS, Thiwan SI, Turner MJ, van Tilburg MA, Gangarosa LM, et al. Contributions of pain sensitivity and colonic motility to IBS symptom severity and predominant bowel habits. Am J Gastroenterol. 2008 Oct;103(10):2550-61. doi: 10.1111/j.15720241.2008.02066.x.

21. Catterall WA, Perez-Reyes E, Snutch TP, Striessnig J. International Union of Pharmacology. XLVIII. Nomenclature and structure-function relationships of voltage-gated calcium channels. Pharmacol Rev. 2005 Dec;57(4):411-25. doi: 10.1124/pr.57.4.5.
22. Kochegarov AA. Pharmacological modulators of voltagegated calcium channels and their therapeutical application. Cell Calcium. 2003 Mar;33(3):145-62. doi: 10.1016/ S0143-4160(02)00239-7.

23. Nowycky MC, Fox AP, Tsien RW. Three types of neuronal calcium channel with different calcium agonist sensitivity. Nature. 1985 Aug 1-7;316(6027):440-3. doi: 10.1038/316440a0.

24. Prior A, Harris SR, Whorwell PJ. Reduction of colonic motility by intravenous nicardipine in irritable bowel syndrome. Gut. 1987 Dec;28(12):1609-12. doi: 10.1136/ gut.28.12.1609.

25. Camilleri M, Boeckxstaens G. Dietary and pharmacological treatment of abdominal pain in IBS. Gut. 2017 May;66(5):966-974. doi: 10.1136/gutjnl-2016-313425.

26. Evangelista S, Cochet P, Bromet N, Criscuoli M, Maggi CA. A distribution study with (14)C-otilonium bromide in the rat: evidence for selective tropism for large intestine after oral administration. Drug Metab Dispos. 2000 Jun;28(6):643-7.

27. Evangelista S, Giachetti A, Chapelain B, Neliat G, Maggi CA. Receptor binding profile of Otilonium bromide. Pharmacol Res. 1998 Aug;38(2):111-7. doi: 10.1006/phrs.1998.0340.

28. Boeckxstaens G, Corazziari ES, Mearin F, Tack J. IBS and the role of otilonium bromide. Int J Colorectal Dis. 2013 Mar;28(3):295-304. doi: 10.1007/s00384-012-1598-0.

29. Clavé P, Acalovschi M, Triantafillidis JK, Uspensky YP, Kalayci C, Shee V, et al. Randomised clinical trial: otilonium bromide improves frequency of abdominal pain, severity of distention and time to relapse in patients with irritable bowel syndrome. Aliment Pharmacol Ther. 2011 Aug;34(4):43242. doi: 10.1111/j.1365-2036.2011.04730.x.

30. D’Arienzo A. D’Agostino L. L'ottilonio bromuro nel trattamento della sindrome del colon irritabile. Rass Int Clin Ter. 1980;60:649-56.

31. Glende M, Morselli-Labate AM, Battaglia G, Evangelista S. Extended analysis of a double-blind, placebo-controlled, 15-week study with otilonium bromide in irritable bowel syndrome. Eur J Gastroenterol Hepatol. 2002 Dec;14(12):1331-8. doi: 10.1097/00042737-20021200000008.

32. Baldi F, Corinaldesi R, Ferrarini F, et al. Clinical and functional evaluation of octilonium bromide in the treatment of irritable bowel syndrome: a double-blind controlled trial. Clin Trials J. 1983;20:77-88.

33. Castiglione F, Daniele B, Mazzacca G. Therapeutic strategy for the irritable bowel syndrome. Ital J Gastroenterol. 1991 Nov;23(8 Suppl 1):53-5.

34. Christen MM-O, Tassignon J-P. Pinaverium bromide: A calcium channel blocker acting selectively on the gastrointestinal tract. Drug Dev Res. 1989 Jan 1;18(2):101-12. doi: $10.1002 /$ ddr.430180202.

35. Evangelista S. Quaternary ammonium derivatives as spasmolytics for irritable bowel syndrome. Curr Pharm Des. 2004;10(28):3561-8. doi: 10.2174/1381612043382972. 
36. Malysz J, Farraway LA, Christen MO, Huizinga JD. Pinaverium acts as L-type calcium channel blocker on smooth muscle of colon. Can J Physiol Pharmacol. 1997 Aug;75(8):969-75. doi: 10.1139/cjpp-75-8-969.

37. Boyer JC, Magous R, Christen MO, Balmes JL, Bali JP. Contraction of human colonic circular smooth muscle cells is inhibited by the calcium channel blocker pinaverium bromide. Cell Calcium. 2001 Jun;29(6):429-38. doi: 10.1054/ ceca.2001.0205.

38. Wittmann T, Fehér A, Rosztóczy A, Jánosi J. [Effectiveness of pinaverium bromide therapy on colonic motility disorders in irritable bowel syndrome]. Orv Hetil. 1999 Feb 28;140(9):469-73.

39. Zheng L, Lai Y, Lu W, Li B, Fan H, Yan Z, et al. Pinaverium Reduces Symptoms of Irritable Bowel Syndrome in a Multicenter, Randomized, Controlled Trial. Clin Gastroenterol Hepatol. 2015 Jul;13(7):1285-1292.e1. doi: 10.1016/j.cgh.2015.01.015.

40. Delmont J. Interet de l'adjonction d'un antispasmodique musculotrope au traitement des constipations douloureuses des colopathies fonctionnelles par le son. Med Chir Dig. 1981;10:365-70.
41. Levy C, Charbonnier A, Cachin M. [Pinaverium bromide and functional colonic disease (double-blind study]. Sem Hop Ther. 1977 Sep-Oct;53(7-8):372-4.

42. Virat J, Hueber D. Colopathy pain and dicetel. Prat Med. 1987;43:32-34. doi: 10.1097/00007890-19870100000008.

43. Hussain Z, Jung DH, Lee YJ, Park H. The Effect of Trimebutine on the Overlap Syndrome Model of Guinea Pigs. J Neurogastroenterol Motil. 2018 Oct 1;24(4):669675. doi: 10.5056/jnm18049.

44. Kang SH, Jeen YT, Koo JS, Koo YS, Kim KO, Kim YS, et al. [Efficacy of fenoverine and trimebutine in the management of irritable bowel syndrome: multicenter randomized double-blind non-inferiority clinical study]. Korean J Gastroenterol. 2013 Nov;62(5):278-87. doi: 10.4166/ kjg.2013.62.5.278.

45. Trimebutine Maleate and Pinaverium Bromide for Irritable Bowel Syndrome: A Review of the Clinical Effectiveness, Safety and Guidelines [Internet]. Ottawa (ON): Canadian Agency for Drugs and Technologies in Health; 2015 Nov 30. 